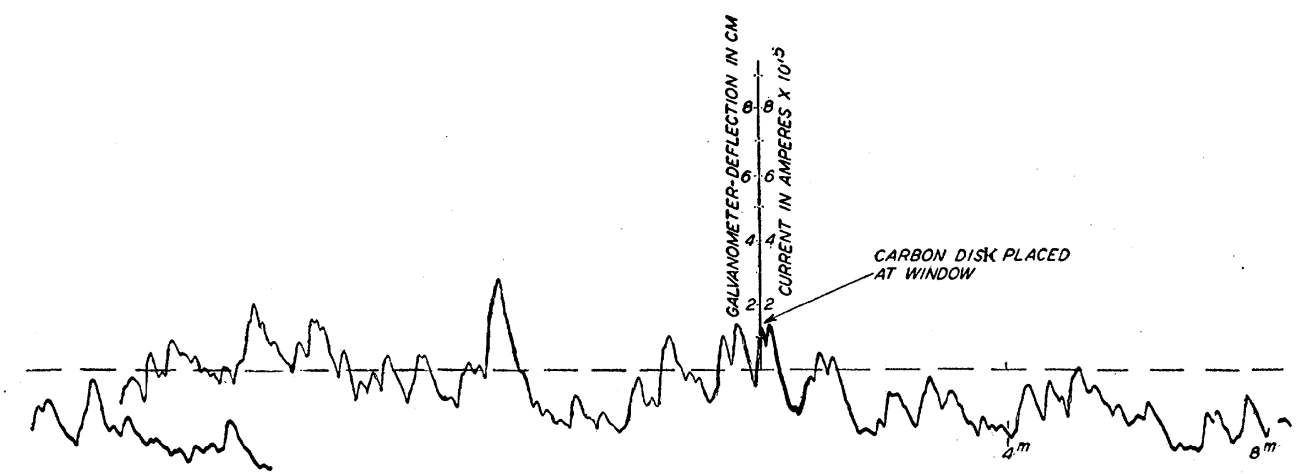

FIG. 2. Radioactivity from protons on carbon.

sensitivity and, in spite of the raggedness of the trace at this extreme sensitivity, a displacement of the mean of about one centimeter could have been detected. This would be one-two thousandth of the effect measured in Fig. 1 and, since the proton-current used was four times the deuton-current, we may conclude that any effect produced by protons on carbon is less than one-eight thousandth of the effect produced by deutons. [Incidentally, this is additional evidence for the extreme purity of our proton-beam after magnetic analysis, as previously reported.]

The wide discrepancy between this result and those reported by the workers in Cambridge and Pasadena does not appear to us to be particularly surprising, since magnetic analysis evidently was not used in their experiments. While it is true that in the experiments at Pasadena residual contamination by deuterium would hardly be expected to amount to 10 percent or 20 percent of the total beam, it is quite possible that the total beam with deuterium flowing might contain only five to ten times as many deutons as with the deuterium-flow cut off. In some of our previous work we determined the yields of several efficient disintegration-processes by working for many days with no deuterium-flow, that is, using only the residue of deuterium left in the tube from previous runs. Further evidence that the percentage of deutons in the Pasadena experiments is much lower than estimated is given by the higher gamm-ray yields from carbon and beryllium we have observed. ${ }^{4}$

We take great pleasure in expressing our gratitude to Professors Urey, Zanetti, and La Mer, of Columbia University, for the supply of deuterium which made these experiments possible.

\section{R. Hafstad}

M. A. Tuve

\section{Department of Terrestrial Magnetism, Carnegie Institution of Washington, Washington, D. C.,} June 1, 1934.

${ }^{4}$ Hafstad, Tuve and Brown, Phys. Rev. 45, 746-747 (1934).

\title{
On the Limitations of the Theory of the Positron
}

In a recent paper ${ }^{1}$ Dirac has suggested a further development of his theory of the positron. Dirac here considers the operators corresponding to charge and current density for a system of electrons in which nearly all the negative energy states are full, and shows that in the presence of an arbitrary external electromagnetic field these operators may be divided into two terms: one of these is infinite, and depends on the field but not on the state of the electrons; the other is finite and determinate, and depends on the field and on the electron state. Dirac makes the suggestion that these second terms be regarded as giving the charge and current density of the electron-positron distribution $(e p d)$ : i.e., that the formalism of his theory of the electron be modified by the subtraction from the operators for charge and current density of the infinite and fielddependent terms. This modification leaves unaltered the Lorentz and gauge invariance of the theory and the validity of the conservation law for charge and current. Because, however, the way in which the operators are to be modified depends upon the value of the electromagnetic field, the method is not readily extended to take account of the field produced by the $e p d$; on the other hand, it gives for the charge and current induced in the $e p d$ by an external field finite and definite results, and thus constitutes in this respect a true theoretical advance.

In the further development of Dirac's suggestions one meets, however, with a curious difficulty, in that it is apparently impossible to find a consistent definition of the operators for the energy and momentum density of the $e p d$. Dirac's density matrix, of course, makes possible a complete formal definition of any operator, which one obtains, in analogy with the methods of the quantum mechanics, by taking the matrix product of the matrix corresponding to the variable in question and the density matrix. This is just what Dirac has done for the current density. If one carries this through for the energy momentum tensor of the $e p d$, one finds in general that its divergence is not given by the Lorentz force with Dirac's expressions for the charge and current. This is because the electromagnetic 
potentials enter explicitly in the density matrix, and lead to the existence of non-Maxwellian forces. With this definition of the dynamical operators, one has thus to abandon either Dirac's expression for the charge and current density or the validity of the conservation laws for energy and momentum. In particular one would not otherwise obtain consistent results, in computing on the one hand the induced charge density, and on the other the polarization energy, of the epd in an electrostatic field.

The simplest way of obviating these difficulties is to modify the density matrix in a way which does not depend on the electromagnetic field strengths present: i.e., to subtract from the operator given by the Dirac theory of the electron the expressions for the state of the electron distribution in the absence of external fields, for which all negative states are full. This procedure leads directly to the theory of the positron as we have developed it. On this theory one finds a polarization of a vacuum by an electromagnetic field which is infinite, and which can only be rendered reasonably unambiguous by special conventions about the way in which the divergent expressions occurring are to be handled. ${ }^{2}$ This theory is therefore not only unable in general to predict the reaction of the $e p d$ to its own field, but can make no unambiguous statements about the fields induced by the $e p d$ under the influence of a given external field.

Nevertheless we believe that these difficulties in no way impair the limited validity of the theory of the positron, a validity which is limited to those questions which do not involve essentially the reaction of the electrons and positrons to their own radiation fields and thus does not extend to problems in which there are external fields whose frequency is of the order of the critical value $m c^{3} / e^{2}$. For at least insofar as the fields are themselves produced by electrons and positrons, the polarization of the epd manifests itself ${ }^{3}$ in effects which are not unambiguously separable from the unknown effects of the radiation reaction of the particles. An instructive illustration of this may be found in the question of the fluctuations of the charge density of the $e p d$, which was brought to our attention by Dr. Bloch. If we consider, for instance, the case of an empty $e p d$ in no external field, for which the expectation value of the charge density vanishes everywhere, we readily find that the expectation value of the square of the charge within any volume is infinite, corresponding to the fact that there are infinite fluctuations in the charge density. If we now ask in what measure it is possible to observe these fluctuations, we see that, to detect them, we must have an observing system (e.g., galvanometer) which will react in accordance with the electron-theoretic laws to electromagnetic fields of arbitrarily high frequency. If we admit that our instruments will not respond to waves of frequency large compared to $m c^{3} / e^{2}$, then we see at once that the observed fluctuations in the charge density will be finite and small, and that the paradoxical predictions of the theory of the positron are quite without physical consequences.

There exists the possibility, which is suggested by classical electron-theory, that we should have in the proton a particle which would respond in accordance with Maxwellian electrodynamic to waves of frequency far greater than $m c^{3} / e^{2}$, and that by its use the effects of the polarization of the epd could be separated from the problems of radiation reaction. There is, however, a growing mass of experimental evidence that so simple a theory of the proton can hardly be correct, and which lends support to the view that the present electrodynamics will be inapplicable in all questions involving lengths of the order of $e^{2} / m c^{2}$. From this point of view the paradoxes of the theory of the positron would be inextricably connected with those of quantum electrodynamics, and the applicability of the two theories would be similarly limited.

It must of course be remarked that the condition that in the field acting upon the charges there be no components of frequency of the order of $m c^{3} / e^{2}$ or greater is a sufficient, but by no means a necessary condition for the applicability of present electrodynamics, for this condition is clearly not relativistically invariant. The necessary condition, as Bohr particularly has emphasized, is that there exist a coordinate system in which such high frequency components are absent, insofar at least as one may consequently neglect the reaction of the charges to their own field. On the other hand, it is not in general possible to infer that, if in a given Lorentz frame high frequency components appear, the reaction of the charges to the low frequency components is correctly given by the Lorentz force. It is for this reason that one need not regard as altogether cogent the arguments recently advanced by v. Weizsaecker ${ }^{4}$ for the validity of the theoretical formulae for the behavior of very high energy radiations in their passage through matter, formulae which are in fact very difficult indeed to reconcile with experiment.

\section{W. H. FURRY*}

J. R. OPPENHEIMER

Pasadena, June 2, 1934.

2 Thus Peierls, to whom we are indebted for telling us of his results, has developed a method by which the polarization of the $e p d$ in an arbitrary electromagnetic may be computed in first order in a gauge and Lorent $z$ invariant manner.

${ }^{3}$ W. H. Furry and J. R. Oppenheimer, Phys. Rev. 45, 245 (1934); pages 261-2.

${ }^{4}$ L. F. v. Weizsaecker, Zeits f. Physik 88, 612 (1934).

* National Research Fellow.

\section{A New Mode of Disintegration Induced by Neutrons}

The capture of a neutron followed by the ejection of an $\alpha$-particle is now a well-known process in the disintegration of light nuclei. Thus in nitrogen this may be written:

$$
{ }_{7} \mathrm{~N}^{14}+{ }_{0} n^{1}={ }_{5} \mathrm{~B}^{11}+{ }_{2} \mathrm{He}^{4} \text {. }
$$

Using as a source of neutrons beryllium bombarded by 3 MV deutons, which were accelerated in the Lawrence-
Livingston ${ }^{1}$ apparatus, I have photographed six examples of a disintegration in which the emitted particle is of smaller charge than an $\alpha$-particle and is probably a proton. The judgment is based on the character of the trace left

${ }^{1}$ E. O. Lawrence and M. S. Livingston, Phys. Rev. 45, 608 (1934). 